\title{
Implications of Metabolically Healthy Obesity-Ganesha Speaks
}

\author{
Rukia Surya Shaikh ${ }^{1}$ Pratiksha Patil ${ }^{2}$ Tejal Lathia ${ }^{3}$ \\ 1Department of Diabetes, Ashirwad Critical Care Hospital, Mulund, \\ Mumbai, India \\ 2Department of Diabetes, Pratham Speciality Clinic, Andheri (East), \\ Mumbai, India \\ ${ }^{3}$ Department of Endocrinology, Apollo and Fortis Hospitals, \\ Navi Mumbai, Maharashtra, India \\ J Soc Health Diab 2019;7:87-88
}

Address for correspondence Tejal Lathia, DM, Department of Endocrinology, Apollo and Fortis Hospitals, Navi Mumbai, Maharashtra 400614, India (e-mail: drtblathia9748@gmail.com).
Abstract
Keywords
- obesity
- metabolic
- diabetes
- hypertension
- cardiovascular disease

The cherubic elephant God Ganesha, a favorite of millions of Hindu devotees, sports a round belly. Humans who have a round belly would be classified as obese. Is all obesity unhealthy? What is metabolically healthy obesity? Different definitions and criteria make the discussion of metabolically healthy obesity difficult and challenging.

\section{Introduction}

The Ganesh or Ganapati festival celebrates the elephant God, the Hindu God of wisdom, knowledge, and power. The festival is held every year in the month of bhadra (mid-August to mid-September). He is the favorite God of millions of Hindus. He uses his powers to solve obstacles (vighna) in the lives of his ardent devotees. Thus, he is also called the vighna-harta (remover of obstacles).

The physical characteristics of Ganesha are very unique-an elephant's head atop a boy's body. He is cherubic with a mischievous smile and a large round belly. The weight does not detract from his ability to defeat his enemies though (asuras-demons).

How does this relate to humans? A human with round cheeks and large belly would be classified as overweight or obese, a disease that afflicts much of humanity.

In India, more than 135 million individuals are affected by obesity. The prevalence varies by age, gender, geographical environment, and socioeconomic status. The ICMR-INDIAB (Indian Council of Medical Research - India Diabetes) study ${ }^{1}$ published in 2015 found the prevalence rate of obesity by body mass index (BMI) to be 12 to $31 \%$ and central obesity by waist circumference (WC) varies from 17 to $36 \%$, respectively.

Obesity is strongly associated with metabolic disorders including diabetes, hypertension, dyslipidemia, cardiovascular disease, and even some cancers. The risk for these disorders appears to start from a BMI as low as $21 \mathrm{~kg} / \mathrm{m}^{2} .{ }^{1}$ Due to this increased risk of morbidity and mortality, obesity is now recognized as a disease in its own right.

Like Ganesha or Ganapati however, 10 to $25 \%$ of obese people and a fraction of morbidly obese individuals are not affected by the expected metabolic consequences related to obesity; this is known as the "metabolically healthy obesity" (MHO). ${ }^{1}$

\section{What Is Metabolically Healthy Obesity?}

Lower liver fat content and lower visceral adipose tissue, greater insulin sensitivity and secretion, less inflammation, greater cardiorespiratory fitness, and predominantly lower body fat deposition are key physiological traits of a metabolically healthy phenotype. MHO is more prevalent in Asian population as compared with Caucasians or multiethnic origin populations. ${ }^{2}$

The National Cholesterol Education Programme (NCEP) criteria require less than 3 of the classic criteria of WC, blood pressure, fasting plasma glucose, triglycerides, high-density lipoprotein cholesterol in patients with BMI $\geq 30 \mathrm{~kg} / \mathrm{m}^{2}$ to qualify as MHO. Other criteria have also included HOMA-IR (Homeostatic model assessment - Insulin Resistance) or some do not consider blood pressure or WC as criteria. ${ }^{3}$

The prevalence and prognostic value of $\mathrm{MHO}$ is controversial and considerably challenging. The lack of standard definition for metabolic health and obesity as well as the dynamic properties of MHO may have contributed to these

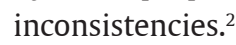

\section{received}

October 7, 2019

accepted after revision

October 24, 2019
DOI https://doi.org/

$10.1055 / \mathrm{s}-0039-3402538$

ISSN 2321-0656. C2019 Novo Nordisk Education
Foundation
License terms

(1) (1) $\Theta \circledast$ 


\section{Implications of MHO}

\section{Type 2 Diabetes}

The risk of type 2 diabetes among individuals with $\mathrm{MHO}$ is controversial. A recent meta-analysis ${ }^{4}$ demonstrated that adult individuals with $\mathrm{MHO}$ were at a more than or equal to four times greater risk of developing type 2 diabetes over time than healthy normal-weight adults, although the risk among healthy obese subjects was approximately half than that of unhealthy obese individuals. Most studies to date have been conducted on Western populations, so it is difficult to generalize these findings to the Asian population. Compared with Caucasians, Asians have a lower BMI but higher risk of developing diabetes for a given BMI. Intuitively, $\mathrm{MHO}$ in Asians would be more likely to have adverse consequences than in Caucasians.

\section{Hypertension}

It has been recently demonstrated ${ }^{4}$ that individuals with MHO had an $~ 1.5$-fold higher association with the incidence of hypertension over a relatively short period of time (median follow-up period, 35.0 months), regardless of the definition used for metabolic health.

\section{Subclinical and Clinical Cardiovascular Disease}

Several reports have assessed subclinical atherosclerosis in individuals with MHO. Khan et $\mathrm{al}^{5}$ found that metabolically healthy overweight or obese middle-aged women had a significantly greater subclinical cardiovascular disease burden, including greater common carotid artery intima media thickness (IMT), aortic pulse wave velocity, and coronary and aortic calcification, compared with normal-weight women.

Similarly, Marini et al ${ }^{6}$ demonstrated that individuals with MHO (defined by the degree of insulin sensitivity using a euglycemic-hyperinsulinemia clamp) had a metabolic and cardiovascular risk profile, including carotid IMT that was intermediate between those of metabolically nonobese subjects and insulin-resistant obese subjects.

Yoo et $\mathrm{al}^{7}$ reported that vascular inflammation, measured using 18F-fluorodeoxyglucose positron emission tomography, was significantly greater in the MHO group compared with the MHNO (metabolically health non-obese) group, although individuals with $\mathrm{MHO}$ had a similar Framingham risk score.

\section{Is There More to Obesity Than Just Diabetes Mellitus and Cardiovascular Diseases?}

According to the World Health Organization, ${ }^{8}$ "overweight and obesity" are defined as abnormal or excessive fat accumulation that may impair health, whereas "health" is defined as a state of complete physical, mental, and social well-being and not merely the absence of disease or infirmity. Therefore, the MHO concept may serve as an example of "metabolically healthy" but does not necessarily mean "healthy" obese.
BMI alone or any anthropometric markers of adiposity do not provide measurements of functionality, quality of life, or other prognostic contextual factors that may further characterize the clinical risk and guide clinical management. Therefore, systematic studies on comorbidities of obesity other than metabolic cardiovascular disorders are necessary to assess more accurately whether MHO individuals are really "healthy."

\section{Wisdom from Ganesha}

Ganesh's large head signifies great wisdom. Using our wisdom, we can learn to make healthy food choices, eat only as much as you need and not more. Eating should be commensurate with activity and age.

Large ears teach us to listen to our body-eat when you are hungry. Instead of following fad diets, eating only when hungry ensures restriction in calorie intake.

Unlike the revered elephant God, humans cannot get away with being metabolically healthy but obese. The burden of complications such as diabetes, hypertension, and subclinical cardiovascular disease should be taken into consideration. As the saying goes "prevention is better than cure"; hence, overweight or obese person treated in time will have better consequences than labeling them as metabolically healthy.

\section{Conflict of Interest}

None declared.

\section{References}

1 Shrivastava U, Misra A, Mohan V, Unnikrishnan R, Bachani D. Obesity, diabetes and cardiovascular diseases in India: public health challenges. Curr Diabetes Rev 2017;13(1):65-80

2 Kim SH, Després J-P, Koh KK. Obesity and cardiovascular disease: friend or foe? Eur Heart J 2016;37(48):3560-3568

3 Velho S, Paccaud F, Waeber G, Vollenweider P, Marques-Vidal P. Metabolically healthy obesity: different prevalences using different criteria. Eur J Clin Nutr 2010;64(10):1043-1051

4 Jung $\mathrm{CH}$, Lee WJ, Song K-H. Metabolically healthy obesity: a friend or foe? Korean J Intern Med (Korean Assoc Intern Med) 2017;32(4):611-621

5 Khan UI, Wang D, Thurston RC, et al. Burden of subclinical cardiovascular disease in "metabolically benign" and "at-risk" overweight and obese women: the Study of Women's Health Across the Nation (SWAN) Atherosclerosis 2011;217(1):179-186

6 Marini MA, Succurro E, Frontoni S, et al. Metabolically healthy but obese women have an intermediate cardiovascular risk profile between healthy nonobese women and obese insulin-resistant women. Diabetes Care 2007;30(8):2145-2147

7 Yoo HJ, Kim S, Hwang SY, et al. Vascular inflammation in metabolically abnormal but normal-weight and metabolically healthy obese individuals analyzed with 18F-fluorodeoxyglucose positron emission tomography. Am J Cardiol 2015;115(4):523-528

8 Obesity and overweight. [cited 2019 Oct 2]. Available at: https://www.who.int/news-room/factsheets/detail/obesityand-overweight 